\title{
Fractal measures of video-recorded trajectories can classify motor subtypes in Parkinson's Disease
}

\author{
Thiago C. Figueiredo ${ }^{a}$, Jamile Vivas ${ }^{b}$, Norberto Peña ${ }^{c}$, José G.V. Miranda ${ }^{a}$ \\ ${ }^{a}$ Physics Institute, Federal University of Bahia, Salvador, Brazil \\ ${ }^{b}$ Faculty of Physiotherapy, University of A Coruña, A Coruña, Spain \\ ${ }^{c}$ Health Sciences Institute, Federal University of Bahia, Salvador, Brazil
}

\begin{abstract}
Parkinson's Disease is one of the most prevalent neurodegenerative diseases in the world and affects millions of individuals worldwide. The clinical criteria for classification of motor subtypes in Parkinson's Disease are subjective and may be misleading when symptoms are not clearly identifiable. A video recording protocol was used to measure hand tremor of 14 individuals with Parkinson's Disease and 7 healthy subjects. A method for motor subtype classification was proposed based on the spectral distribution of the movement and compared with the existing clinical criteria. Box-counting dimension and Hurst Exponent calculated from the trajectories were used as the relevant measures for the statistical tests. The classification based on the powerspectrum is shown to be well suited to separate patients with and without tremor from healthy subjects and could provide clinicians with a tool to aid in the diagnosis of patients in an early stage of the disease.
\end{abstract}

Keywords

Fractal dimension; Hurst exponent; Parkinson's Disease; Motor subtypes 


\section{Introduction}

In the past few years, the development of new technologies has provided different methods to quantify objectively the extent and the characteristics of the motor symptoms that are present in Parkinson's Disease. The aim of this innovation effort is to provide quantitative measured data that can be used by professionals in the health sciences to support clinical diagnosis. These quantities can be combined with the subjective functional clinical scales such as the Hoehn \& Yahr scale (H\&Y) [1], or the Unified Parkinson's Disease Rating Scale (UPDRS) [2] to provide further information on the disease.

Parkinson's Disease is a neurodegenerative disease characterized by many motor symptoms such as slowness of movements, rest tremor (4-7 Hz), postural instability, neuromuscular rigidity, difficulty to swallow and severe functional incapability in its later stages [3] ; [4]. Its symptoms, despite being known since the 19th century [5], are still not fully understood and there are many different physiological models to explain the generation and the wide variability of their expression [6].

The existence of two different motor subtypes has been recently considered in the literature in an attempt to account for this symptom variability [7] ; [8]. One motor subtype presenting tremor as the main symptom was defined as the Tremor Dominant (TD) subtype, while the Posture Impairment/Gait Difficulty (PIGD) subtype presented posture related impairments without tremor [9]. Other subtypes have been identified by clustering analysis methods, such as the Young Onset (YOPD) and Late Onset (LOPD) subtypes [10] ; [11]. It is already known that Parkinson's Disease is a condition with multiple causes [12] and these different subtypes might be associated with different causes and different physiological mechanisms within the disease. Another possibility is that these subtypes could be associated with different stages of the disease [13]. This work focuses on TD and PIGD subtype classification, for their major prevalence and for the direct relation they bear with motor measurements.

The majority of quantitative experimental methods of assessment for tremor measurement has been based on indirect time-series measurements generated from accelerometry [14] ; [15] and electromyography [16] ; [17], as reviewed by Ref. [18]. Both these indirect methods are subject to noise from important sources, such as numerical integration and mechanical noise in the case of accelerometry [19] and background neuromuscular activity in the case of electromyography [20]. Furthermore, the studies that encompassed electromyographic measures of tremor in Parkinson's Disease did not address the issue of variability inside the disease, with the presence of different subtypes [21]; [8] ; [22]. An interesting recent study [23] have used spirogram tests with touch screen sensors and has been able to establish the frequency and amplitude of tremor and the correlation of these measures with well known tremor rating scales. Yet the need for new and precise quantitative assessment tools that can assist clinical diagnosis is still a matter of relevance in the context of Parkinson's Disease.

The data processing methods used for the evaluation of the symptoms of the disease are perhaps as important as the experimental procedures used to register them. The use of nonlinear measurements is a natural possibility, considering the nonlinear and variable nature of biological signals. These methods can also provide important information in situations where regular timefrequency analysis alone is not sufficient to describe the studied behavior.

In this regard, the Hurst exponent $\mathrm{HH}$ [24] is a statistical index that quantifies temporal correlations in a time-series and is widely used in areas such as hydrology, time-series and stock market analysis [25]. It can take values between 0 and 1 . For $0<\mathrm{H}<0.50<\mathrm{H}<0.5$, the fluctuations are said to be anti-persistent, implying that a period of growth is soon followed by a period of decrease, and vice versa. Anti-persistent correlations are often related to negative feedback control mechanisms, extensively found on regulation mechanisms in the human body [26] ; [27]. For $0.5<\mathrm{H}<10.5<\mathrm{H}<1$, the correlations are said to be persistent. A period of growth is likely to be 
followed by another period of growth and a period of decrease is likely to be followed by another period of decrease. Hence the index $\mathrm{HH}$ provides a method to evaluate statistical fluctuations in a time-series. Complex systems such as human biological signals and the climate often do not present a single value for the Hurst index, but instead present different scaling relationships in different time scales, associated with different phenomena. Specifically, stochastic series modulated by periodical oscillations present a decreasing region in slope that is associated with the wavelength of the periodic signal [28] ; [29].

Another important measure used in biological signals analysis is the Box-Counting dimension [30]. The box-counting dimension DD of a given two-dimensional trajectory provides a measure of the roughness or irregularity of the profile and, perhaps more importantly, the occupation pattern of space by the trajectory being considered. The value of DD can vary between 0 and 2 in a plane, reflecting the way that a given structure fills the space in which it is embedded. It has been used to describe irregular structure that appears in a wide range of natural systems [31] ; [32] and most notably in human physiology [33]. As will be discussed throughout this work, both these nonlinear measurements can be applied to the study of human movement with important results.

\section{Methods}

The experimental procedures conducted in this work were approved by the research ethics committee of the Professor Edgar Santos University Hospital of the Federal University of Bahia and registered under the number 118/2012, in accordance with the Declaration of Helsinki (1964). All participants signed a written informed consent term.

The experimental procedure aimed to test the existence of measurable features that could, under the same conditions, separate control individuals from TD and PIGD motor subtypes, considering the intersubject variability of symptom expression. It also intended to test for measurable features that could separate controls from individuals with Parkinson's disease.

\subsection{Patients}

The trials involved 7 healthy control individuals, in a group of 4 women (mean age 72.5 [min 63, max 81]) and 3 men (mean age 68[min 67, max 70]), and 14 patients in a group of 6 women (mean age 67.17[min 58, max 79]) and 8 men (mean age 74.12[min 67, max 84]) with idiopathic Parkinson's Disease enrolled in the Parkinson Ferrol Association (Galicia, Spain). All patients were receiving medical and physical therapeutic treatment. All the patients were in the active (on) phase of the medication, as for the moment of the data acquisition. None of the patients had been subject to surgical intervention, for the moment of the experiment. Patients were initially classified within the two motor subtypes (TD/PIGD), according to the clinical criterion presented by Stebbins and coworkers [10], based on the UPDRS scale. In this classification, three of the patients were classified under the TD subtype, while the other 11 patients were classified under the PIGD subtype.

\subsection{Procedures}

In order to evaluate the properties of tremor and the proposed hypotheses, the movement of the hand of the 21 subjects was filmed using high-speed cameras (Casio FZ100, $120 \mathrm{fps}, 720 \mathrm{p}$ ). The individuals were filmed in the upright position, with the arms free to move in the sagittal plane and the back leaned against a wall. The camera was positioned at a fixed distance from the subject to record movement in the anteroposterior plane (Fig. 1). 


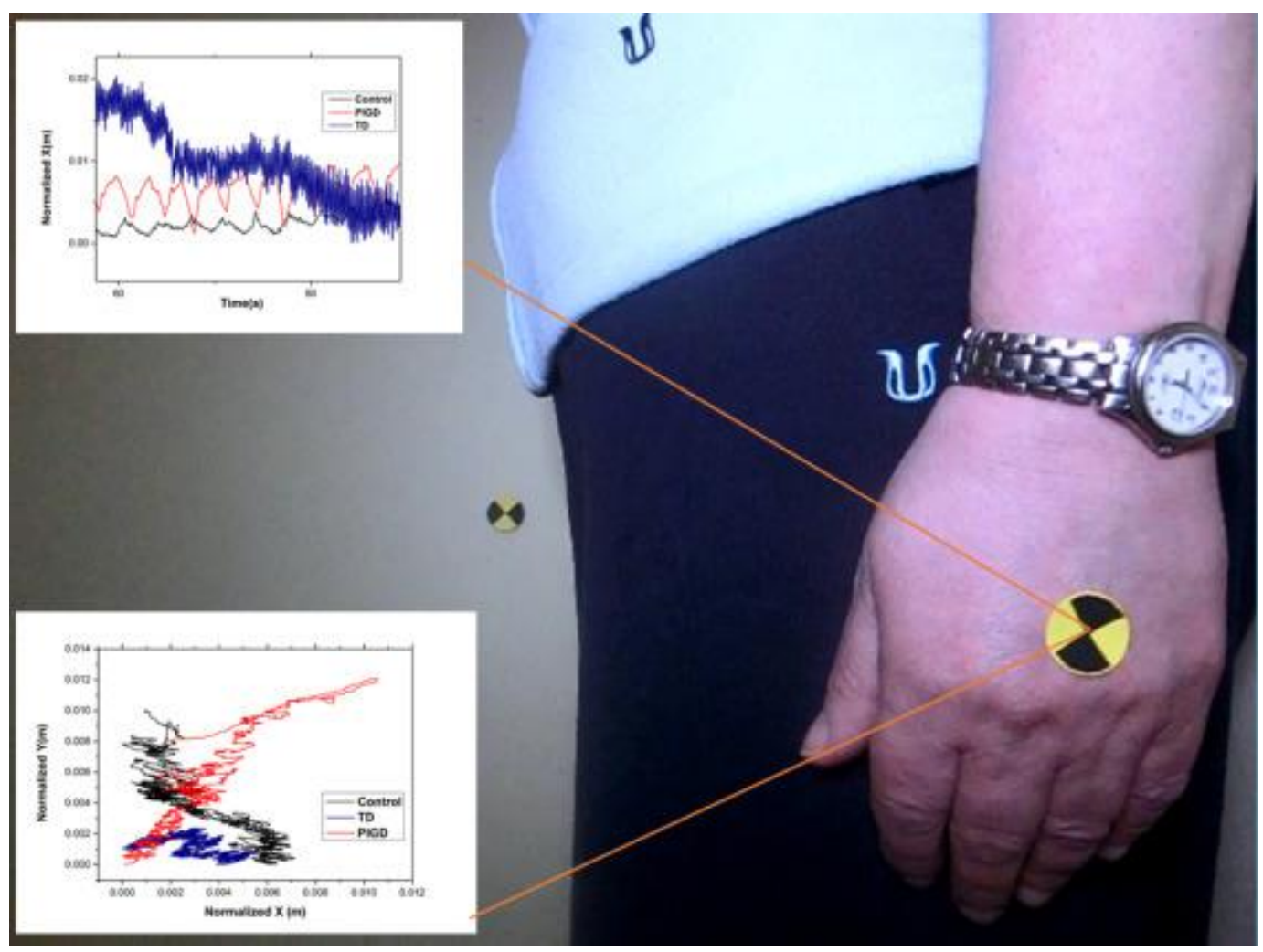

Fig. 1. Subject in the upright position with the back against a wall. A point on the wall was also marked, to evaluate light fluctuations.

\subsection{Data analysis}

\subsubsection{CVMob}

After recording, the video files were loaded into a software called CVMob for the extraction of the time-series. CVMob is a computational vision software that uses optical flow measurements to track the motion of a point in the video [34] ; [35]. The software provides kinematic measures for the selected points in the video such as position, velocity, acceleration and angles (see supplementary material, Appendix A). In the upper (lower) left corner of Fig. 1 are represented examples of position time-series and configuration-space trajectories obtained from the CVMob software.

\subsubsection{Time-series and trajectory}

The position time-series of the point in the hand of the subjects was used to calculate the amplitude of tremor for all participants, but it presented no significant differences between any of the groups studied, as will be detailed in the discussion section. The position series was also used to calculate the Hurst exponent with the Root Mean Square (RMS) method [36]. The RMS method consists in estimating the average standard deviation of the signal for different scales with the expression:

$\operatorname{RMS}(\epsilon)=\frac{1}{N-\epsilon} \sum_{\epsilon=1}^{N-1}\left[\frac{1}{\epsilon} \sum_{i=1}^{\epsilon}\left(X\left(t_{i}\right)-X_{\epsilon}\right)^{2}\right]^{\frac{1}{2}}$ 
where $X(t)$ is the time-series, $X \bar{\epsilon}$ the average value of the signal in a window of size and $N$ the total number of measures.

Representative results of the RMS curve can be seen in Fig. 2. The results of $H$ obtained in this study were calculated by applying linear regression to the $\log -\log$ plot of the RMS curve. The scale region used to fit the RMS curve was between 0.14 and $0.25 \mathrm{~s}$. This scale region is associated with the frequency of tremor $(4-7 \mathrm{~Hz})$.

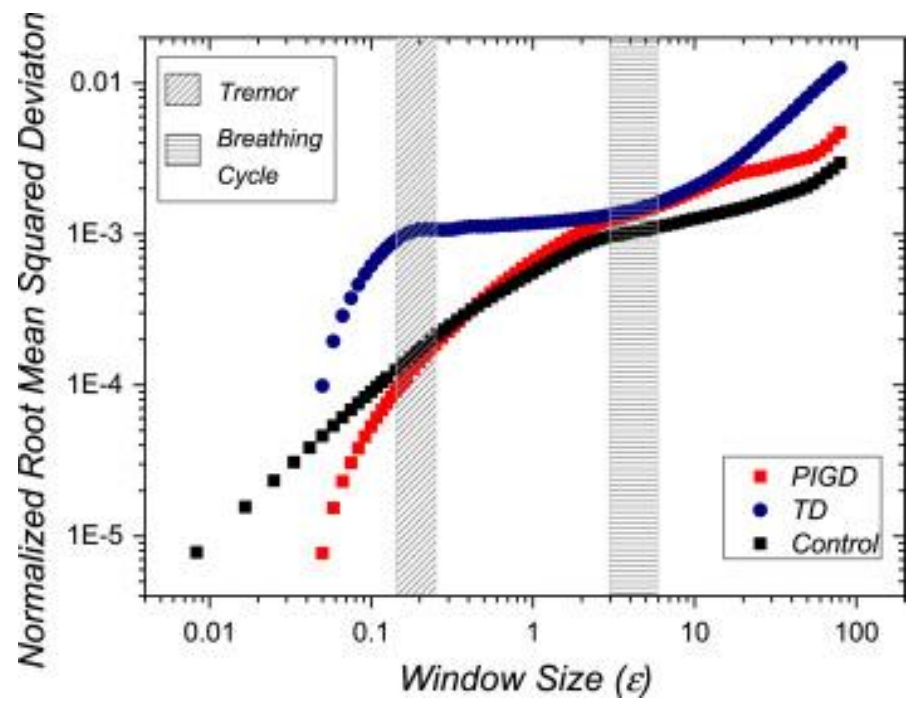

Fig. 2. Representative curves of the RMS method applied to Control, TD and PIGD subjects. The intermediate region between 0.14 and $0.25 \mathrm{~s}$ region of the graph was used to test for statistical differences between subjects, associated with the frequency of tremor.

The fractal dimension $D$ of the trajectory in the $\mathrm{X}-\mathrm{Y}$ plane was also calculated, using the BoxCounting method [30]. For 2-dimensional profile such as the movement on a plane, the fractal dimension can assume any value between 1 and 2, with 1 being a straight line trajectory and 2 being a completely filled plane. The different values of $D$ between 1 and 2 express the occupation of the 2-D space by the trajectory and are related to the irregularity of the profile.

The results of the Hurst index and the fractal dimension were then compared using the MannWhitney statistical test.

\subsubsection{Subject reclassification}

After processing of the acquired hand position time-series, it was verified that part of the subjects presented peaks in the Power Spectral Density (PSD) function for the hand trajectories, which is a characteristic behavior presented by signals with oscillatory behavior. Furthermore, the data from these subjects presented a peak located within the particular frequency region of parkinsonian tremor $(4-7 \mathrm{~Hz})$. Subjects were then reclassified within the two subtypes, according to the following criterion: Subjects presenting a peak in the PSD within the range of 4-7 Hz were classified as Tremor Dominant subtype, while those that did not present such a peak were classified as Posture Impairment/Gait Difficulty subtype (Fig. 3). Under this new criterion, 5 patients were classified under the TD subtypes, while 9 patients were classified as PIGD subtype. 
Both classifications are presented in Table 1. Statistical analysis was then carried on using this new classification criterion and compared with the initial classification.

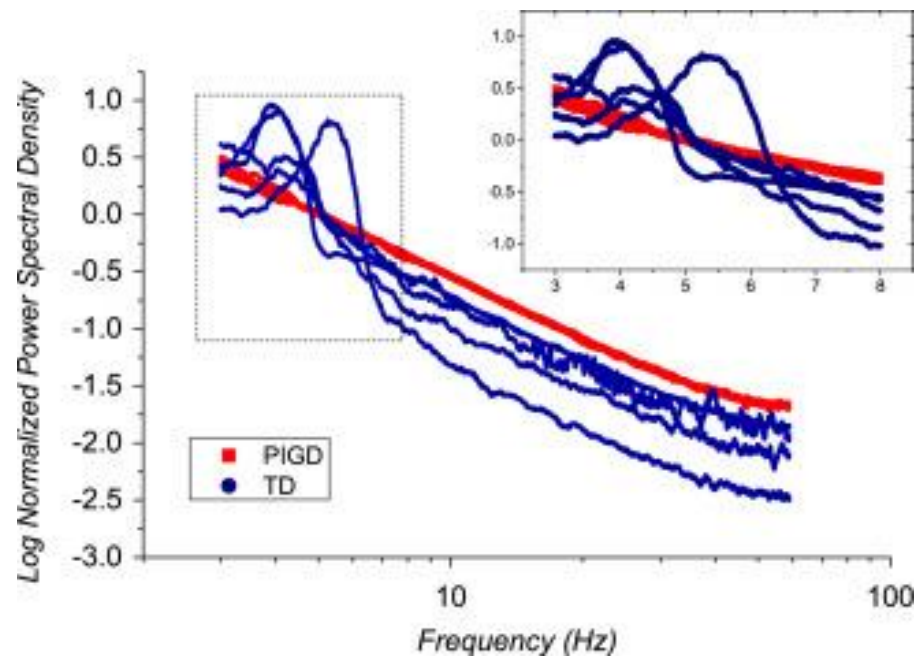

Fig. 3. Power Spectral Density for individuals reclassified as TD subtype (blue) and PIGD (red). The TD signals present peaks in the PSD within the frequency of tremor $(4-7 \mathrm{~Hz})$. (For interpretation of the references to colour in this figure legend, the reader is referred to the web version of this article.)

Table 1. Classification of subjects under the UPDRS and the proposed Power Spectrum criteria.

\begin{tabular}{lll}
\hline Subject & UPDRS criterion & Power spectrum criterion \\
\hline & & \\
P1 & PIGD & PIGD \\
P2 & PIGD & PIGD \\
P3 & TD & TD \\
P4 & PIGD & PIGD \\
P5 & PIGD & PIGD \\
P6 & PIGD & PIGD \\
P7 & PIGD & TD \\
P8 & PIGD & PIGD \\
P9 & PIGD & PIGD \\
P10 & PIGD & TD \\
P11 & PIGD & PIGD \\
P12 & PIGD & TD \\
P13 & TD & TD \\
P14 & TD & PIGD \\
Controls & & 7 \\
& & \\
\hline
\end{tabular}




\section{Results}

The Hurst exponent $H$ was estimated for time-series of horizontal direction movement of tremor. The results presented no significant differences between TD and PIGD groups using the initial clinical classification criterion (Fig. 4(a)) with a $p$-value $p=0.876$, nor in the comparison between TD and control $(p=0.494)$ and PIGD and control $(p=0.717)$. For the power-spectrum criterion (Fig. 4(b)), the Hurst exponent was significantly higher for PIGD subjects, with $p<0.01$. The differences between TD and control were also significant with $p<0.01$. The differences between PIGD and control groups were not significant $(p=0.057)$.
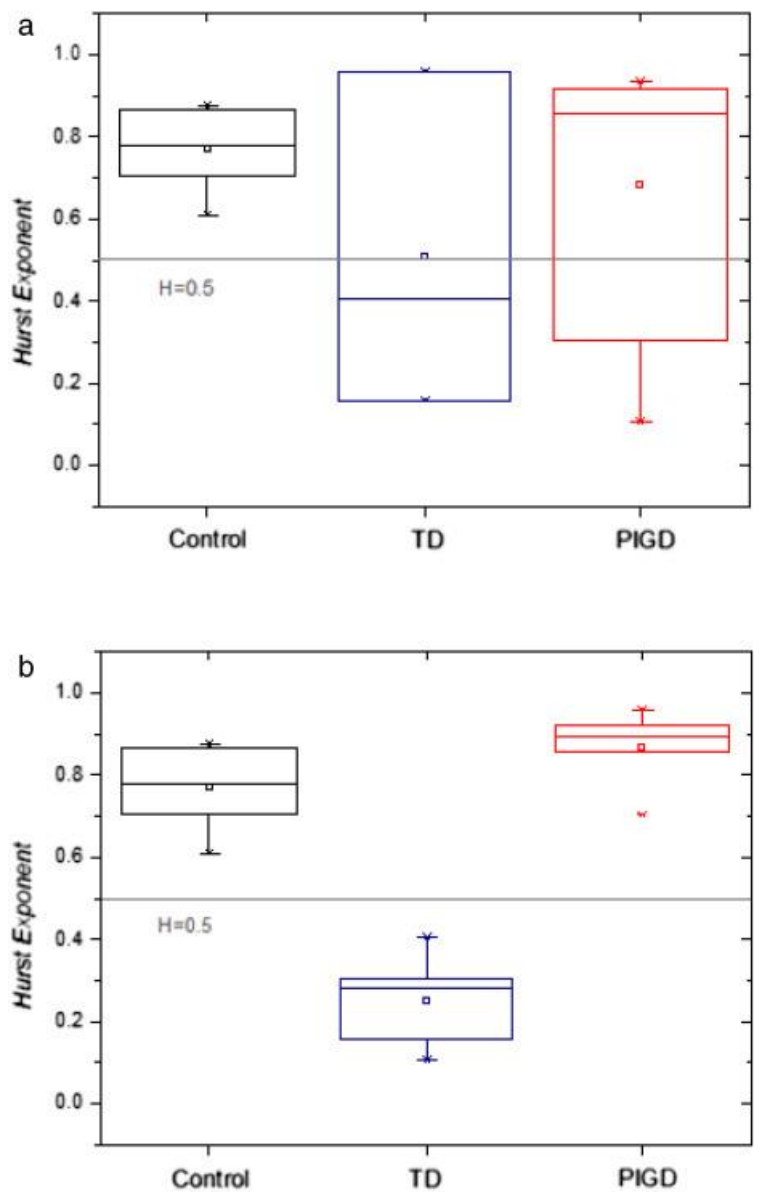

Fig. 4. (a) Results of the Hurst Exponent using the clinical classification criterion of Ref. [10]. (b) Results of $H$ using the power-spectrum criterion.

For the fractal dimension $D$ of the trajectory of the tremor, measured under the clinical criterion (Fig. 5(a)), the differences between TD and PIGD were not significant, with $p=0.35$. The differences between the value of $D$ for PIGD patients and controls presented significant differences with $p=0.023$, while the differences between TD and controls did not $(p=0.65)$. Using the power-spectrum criterion (Fig. 5(b)), the differences between TD and PIGD were also not significant, with $p=0.689$. The values of $D$ were also higher for PIGD patients then controls, with $p=0.044$, while the differences between TD and controls were not significant $(p=0.189)$. 

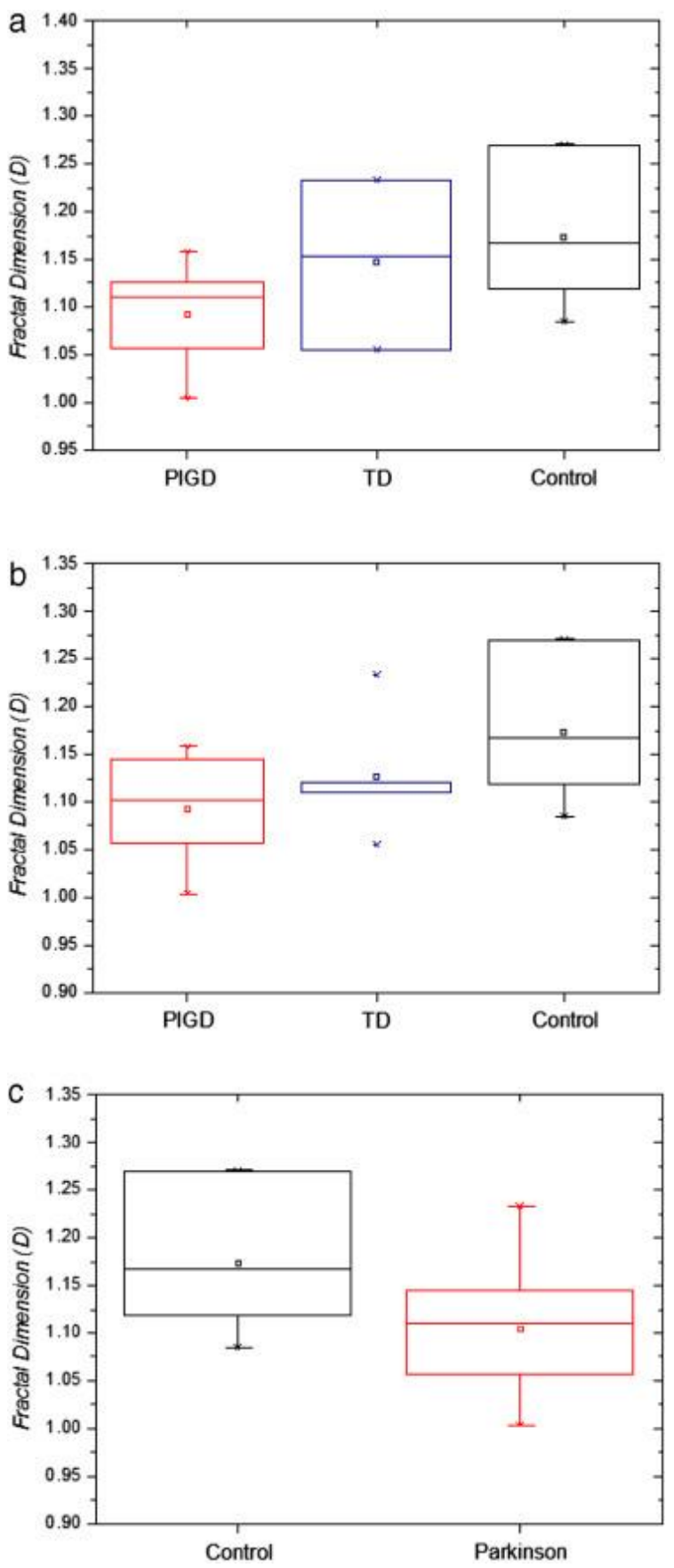

Fig. 5. (a) Results of the fractal dimension using the clinical classification criterion of Ref. [10]. (b) Results of $D$ using the power-spectrum criterion. (c) Comparison of the fractal dimension between Parkinson and control groups, regardless of motor subtype. 
When individuals were analyzed into patient and control groups, without reference to motor subtypes, the value of fractal dimension were significantly smaller in the Parkinson group with a $p$-value $p=0.04$ (Fig. 5(c)). A summary of the statistical results is presented in Table 2, along with the effect sizes measured by Cohen's $d$ index. Cohen's $d$ is a complementary assessment of significance of results that measures how big the statistical effect is, taking into account the size of the tested samples [37]; [38]. Values of $d$ are conventionally interpreted as small $(d>0.2)$, medium $(d>0.5)$ and large $(d>0.8)$ effects. Some authors also include a fourth level for very large effects $(d>1.3)$.

Table 2. Results of hypotheses testing and corresponding effect strength (measured by Cohen's $d$ coefficient).

\begin{tabular}{|c|c|c|c|c|}
\hline $\begin{array}{l}\text { Unequal sample size's Mann- } \\
\text { Whitney test }\end{array}$ & UPDRS criterion & Cohen's $d$ & Power spectrum criterion & Cohen's $d$ \\
\hline $\mathrm{H}($ Control × PIGD $)$ & $p=0.717$ & 0.376 & $p=0.057$ & 1.078 \\
\hline $\mathrm{H}($ Control × TD $)$ & $p=0.494$ & 0.881 & $p<0.01$ & 4.881 \\
\hline $\mathrm{H}(\mathrm{PIGD} \times \mathrm{TD})$ & $p=0.876$ & 0.491 & $p<0.01$ & 5.867 \\
\hline D $($ Control × PIGD $)$ & $p<0.05$ & 1.31 & $p<0.05$ & 1.244 \\
\hline $\mathrm{D}($ Control $\times \mathrm{TD})$ & $p=0.65$ & 0.327 & $p=0.189$ & 0.694 \\
\hline $\mathrm{D}(\mathrm{PIGD} \times \mathrm{TD})$ & $p=0.35$ & 0.754 & $p=0.689$ & 0.542 \\
\hline $\mathrm{D}(\mathrm{PD} \times$ Control $)$ & $p<0.05$ & & 1.042 & \\
\hline
\end{tabular}

\section{Discussion}

The amplitude of tremor, which at first appeared as an important information to distinguish between controls and patients with different subtypes of disease presented no useful information in this sense. Neither unfiltered position time-series nor $4-\mathrm{Hz}$ high-pass filtered data presented statistically significant differences between any conceivable group. This effect is due to the great intersubject variability found in human biological signals and in particular in human movement.

Concerning the reclassification process, it is worth noting the fact that some patients clinically classified as tremor dominant did not present tremor in the power-spectrum density, while others classified as not presenting tremor did so in the spectral analysis (Table 1). Different motor expressions of the disease are in general associated with different rehabilitation strategies and the development of an objective and simple tool to separate clinical subtypes can be of great use in this decision process, especially in early stages of the disease.

The power spectrum criterion combined with the Hurst exponent was able to successfully separate posture impairment and control subjects from tremor dominant subjects, with large effect size (Table 2, Fig. 4). The effect size for the comparison between PIGD and control subjects was also considerably large, despite the fact that the differences were not significant at a confidence level of $95 \%$. Using the UPDRS criterion however, the effect sizes were small and the statistical differences not significant.

For the fractal dimension of the trajectory of the tremor, the effect sizes were small in both criteria and all tests, except when comparing PIGD subjects with control subjects. In this case the effects were larger and the statistical differences significant. This indicates that the statistical significance of the comparison of subjects with Parkinson's Disease and controls is most likely due to the PIGD patients. In turn, this result corroborates the hypothesis that PD patients, even in the absence of tremor, present different patterns of movement irregularity when compared with 
healthy subjects. The results imply that this difference can be adequately measured by the fractal dimension.

Previous studies evaluated gait and balance [39] and physiological hand tremor [40] in Parkinson's Disease, both using fractal dimension of time-series as the characterization parameter. The authors uses time-series of finger tremor (with piezoelectric sensors) [40] and accelerometer raw data [39]. One way to compare these previous studies with our results is to use the relation between the calculated Hurst exponent and its corresponding fractal dimension. For self-affine processes, the local properties given by the fractal dimension and the long range correlations represented by the Hurst exponent are related by the following expression:

$D+H=n+1$

where $n$ is the number of dimensions of the underlying process. For a unidimensional time-series, we have $D=2-H$, which was used in this study.

The values of $D$ for the time-series of tremor are shown in Fig. 6 and compared with the value of the fractal dimension calculated with the box-counting method, under the power-spectrum criterion presented in this work. In Fig. 6, we can notice a clustering behavior, where the points corresponding to the TD patients are far apart from the other two groups. The distance between the PIGD and the control clusters is smaller and this distinction is harder to perceive.

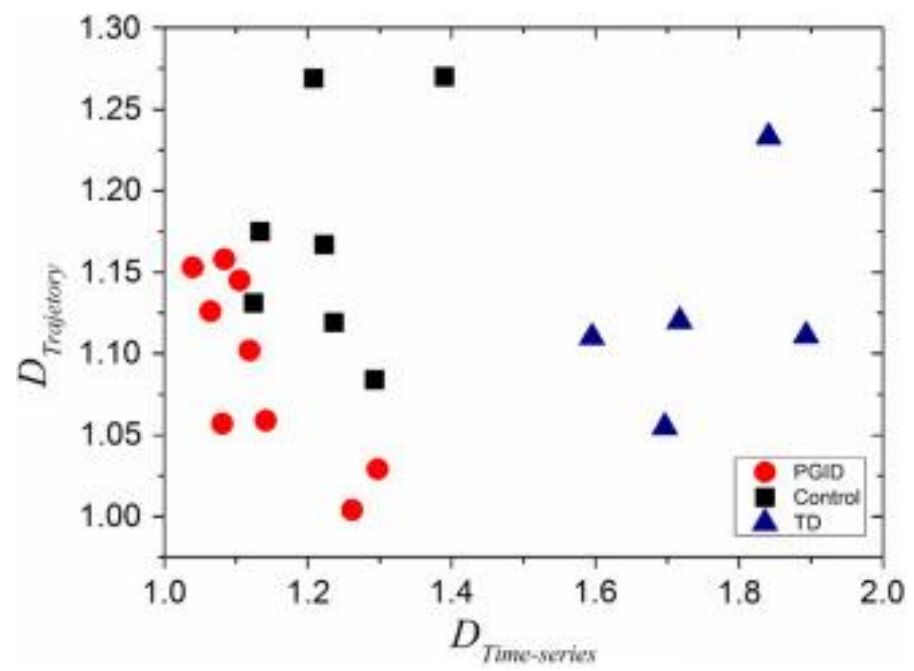

Fig. 6. A plot of the fractal dimension calculated from the Hurst exponent versus the fractal dimension calculated from the Box-Counting method, for the spectrum criterion.

Both previous works also found higher values of $D$ for the PD subjects when compared with controls, with $D$ values similar to those found in this study. It is important to notice that three studies, using different measurements with different techniques, reached similar results, all indicating that PD subjects have less correlated movements than healthy subjects. 
A recent study that aimed to objectively characterize motor differences between PIGD and TD subtypes in Parkinson's Disease used lumbar tri-axial accelerometers to evaluate a different aspect of the quality of life of patients, namely gait and balance [41]. In our study, we were able to separate the subjects in the same subgroups using a simpler procedure, with the use of a camera and a computer for offline data processing.

Hausdorff and coworkers [42] also studied autocorrelations separately in the context of aging and Huntington's disease, in comparison with young healthy subjects. In both cases, they have found a smaller Hurst exponent in comparison with the control subjects, a tendency of more random movements $(H \rightarrow 0.5)$. It is important to observe that they have calculated the Hurst exponent through the detrended fluctuation analysis method, which is not strictly the same as the Hurst exponent (it is not strictly smaller than 1). That aside, their result points to more irregular movements, when compared to young healthy individuals. Our results do confirm this behavior, with patients showing generally more random movements (although the trends are different within the two subtypes). Parkinson's disease subjects present small amplitude trajectories that are supposedly related to the inhibitory mechanisms of the disease [6].

All the previous works evaluated the temporal dynamics of the fluctuations using the Hurst exponent of the time-series. In this work, the fractal geometry of the trajectory in the $\mathrm{X}-\mathrm{Y}$ plane was also calculated, which in turn is a measure of the spatial dynamics of tremor. In this spatial evaluation of the fluctuation patterns, the values of $D$ present the opposite trend when compared to the temporal dynamics, with lower values of $D$ for Parkinson subjects. This implies that the dynamics of tremor in time may present different structure and fluctuation patterns, when compared with its spatial dynamics.

Future theoretical models aiming to find relationships between the fluctuation patterns of the time-series along the spatial directions and the trajectory they form in three-dimensional space could contribute to a better understanding of how different feedback systems are related in Parkinson's Disease.

The results obtained in this work, although still preliminary, point to the possibility of using the protocol described in this work for diagnosis in clinical practice, as well as to evaluate different aspects of tremor in Parkinson's Disease. Future studies should be conducted to explore the effectiveness of the protocol in clinical practice. The correlations between nonlinear measures and functional scales that describe the evolution of the disease should also be addressed. This work corroborates the use of new technologies as tools for objectively measuring the symptoms of the disease, as this can in turn provide robustness to the clinical scales such as Hoehn-Yahr or UPDRS scales. Furthermore, the proposed measurement protocol is simple and can be readily applied in clinical practice with inexpensive equipment. The use of nonlinear quantities to characterize the movement of patients, together with usual time-frequency measurements, is also recommended, as both tools can provide complementary information for the study of human motor behavior and its changes in disease.

\section{Acknowledgments}

The authors would like to acknowledge the Parkinson Ferrol Association in Spain, for providing the data from the patients. This work received financial support from the National Council for Technical and Scientific Development (CNPq), grant number 306571/2011-0, and research project number 481414/2012-6. This work received also financial support from the Coordination of Improvement of Higher Education Personnel (CAPES) in the form of a student scholarship.

\section{Appendix A. Supplementary material}

The following is the Supplementary material related to this article. 


\section{References}

[1]. M. Hoehn, M. Yahr. Parkinsonism: onset, progression and mortality. Neurology, 17 (5) (1967), pp. $427-$ 442.

[2]. C.G. Goetz, et al. Movement Disorder Society-sponsored revision of the Unified Parkinson's Disease Rating Scale (MDS-UPDRS): Scale presentation and clinimetric testing results. Mov. Disord., 23 (15) (2008), pp. 2129-2170.

[3]. E. Tolosa, G. Wenning, W. Poewe. The diagnosis of Parkinson's disease. Lancet Neurol., 5 (1) (2006), pp. $75-86$.

[4]. J.A. Obeso, M.C. Rodríguez-Oroz, M. Rodríguez, J.L. Lanciego, J. Artieda, N. Gonzalo, C.W. Olanow. Pathophysiology of the basal ganglia in Parkinson's disease. Trends Neurosci., 23 (10-Suppl) (2000), pp. S8-S19.

[5]. J. Parkinson. An Essay On The Shaking Palsy. Wittingham and Rowland, Londres (1817).

[6]. T. Wichmann, M.R. Delong. Neurocircuitry of Parkinson's disease. K.L. Davis, D. Charney, J.T. Coyle, C. Nemeroff (Eds.), Neuropsychopharmacology: The Fifth Generation of Progress, Williams and Wilkins (2002), pp. 1761-1779.

[7]. M. Selikhova, D.R. Williams, P.A. Kempster, J.L. Holton, T. Revesz, A.J. Lees. A clinico-pathological study of subtypes in Parkinson's disease. Brain, 132 (2009), pp. 2947-2957.

[8]. S. Morrison, G. Kerr, K.M. Newell. Differential time- and frequency-dependent structure of postural sway and finger tremor in Parkinson's disease. Neurosci. Lett., 443 (3) (2008), pp. 123-128.

[9]. W.J. Zetusky, J. Jankovic, F.J. Pirozzolo. The heterogeneity of Parkinson's disease: Clinical and prognostic implications. Neurology, 35 (4) (1985), p. 522.

[10]. G.T. Stebbins, C.G. Goetz, D.J. Burn, J. Jankovic, T.K. Khoo, B.C. Tilley. How to identify tremor dominant and postural instability/gait difficulty groups with the movement disorder society unified Parkinson's disease rating scale: Comparison with the unified Parkinson's disease rating scale. Mov. Disorders, 28 (5) (2013), pp. 668-670.

[11]. M.A. Thenganatt, J. Jankovic. Parkinson disease subtypes. JAMA Neurol., 71 (4) (2014), pp. 499-504.

[12]. C. Colosimo, M. Stacy. Is Parkinson's Disease Genetic?. Mov. Along: A Mov. Disord. Soc. Update, 14 (1) (2010), pp. 1-11.

[13]. G. Alves, J.P. Larsen, M. Emre, T. Wentzel-Larsen, D. Aarsland. Changes in motor subtype and risk for incident dementia in Parkinson's disease. Mov. Disorders, 21 (8) (2006), pp. 1123-1130.

[14]. K.C. Veluvolu, W.T. Ang. Estimation of physiological tremor from accelerometers for real-time applications. Sensors (Basel, Switzerland), 11 (3) (2011), pp. 3020-3036.

[15]. S.J.G. Lewis, T. Foltynie, A.D. Blackwell, T.W. Robbins, A.M. Owen, R.A. Barker. Heterogeneity of Parkinson's disease in the early clinical stages using a data driven approach. J. Neurol. Neurosurg. Psychiatry, 76 (3) (2005), pp. 343-348.

[16]. A.I. Meigal, S. Rissanen, M.P. Tarvainen, P.A. Karjalainen, I.A. Iudina-Vassel, O. Airaksinen, M. Kankaanpää. Novel parameters of surface EMG in patients with Parkinson's disease and healthy young and old controls. J. Electromyography Kinesiol., 19 (3) (2009), pp. e206-e213.

[17]. P.E. O’Suilleabhain, J.Y. Matsumoto. Time-frequency analysis of tremors. Brain, 121 (1998), pp. $2127-$ 2134.

[18]. P.H. Mansur. A review on techniques for tremor recording and quantification. Crit. Rev. Biomed. Eng., 35 (5) (2007), pp. 343-362.

[19]. F.A. Levinzon. Fundamental noise limit of piezoelectric accelerometer. IEEE Sens. J., 4 (1) (2004), pp. 108-111.

[20]. E.A. Clancy, E.L. Morin, R. Merletti. Sampling, noise-reduction and amplitude estimation issues in surface electromyography. J. Electromyography Kinesiol., 12 (1) (2002), pp. 1-16.

[21]. L. Timmermann, J. Gross, M. Dirks, J. Volkmann, H.J. Freund, A. Schnitzler. The cerebral oscillatory network of parkinsonian resting tremor. Brain, 126 (1) (2002), pp. 199-212.

[22]. K.P. Roland, G.R. Jones, J.M. Jakobi. Parkinson's disease and sex-related differences in electromyography during daily life. J. Electromyography Kinesiol., 23 (4) (2013), pp. 958-965.

[23]. R.J. Elble, et al. Tremor amplitude is logarithmically related to 4-and 5-point tremor rating scales. Brain, 129 (10) (2006), pp. 2660-2666.

[24]. H.E. Hurst. Long-term storage capacity of reservoirs. Trans. Amer. Soc. Civ. Eng. (1951), pp. 116-770.

[25]. R. Costa, G.R. Vasconcelos. Long-range correlations and nonstationarity in the Brazilian stock market. Physica A, 329 (2003), pp. 231-248.

[26]. L. Sherwood. Human Physiology: From Cells to Systems. Cengage Learning (2008).

[27]. J.E. Hall. Guyton and Hall Textbook of Medical Physiology: Enhanced E-book. Elsevier Health Sciences (2010).

[28]. J.G.V. Miranda, R.F.S. Andrade. R/S analysis of pluviometric records: comparison with numerical experiments. Physica A, 295 (2001), pp. 38-41. 
[29]. J.G.V. Miranda, R.F.S. Andrade, A.B. Silva, C.S. Ferreira, A.P. Gonzalez, J.L.C. Lopez. Temporal and spatial persistence in rainfall records from Northeast Brazil and Galicia. Theor. Appl. Climatol., 77 (2004), pp. 113-121.

[30]. B.B. Mandelbrot. The Fractal Geometry of Nature. W. H. Freeman and Co (1982).

[31]. D.L. Turcotte. Fractals and Chaos in Geology and Geophysics. Cambridge University Press, Cambridge (1997).

[32]. R.F. Voss. Random fractals: Self-affinity in noise, music, mountains, and clouds. Physica D, 38 (1989), pp. 362-371.

[33]. J.B. Bassingthwaighte, L.S. Liebovitch, B.J. West. Fractal Physiology. Oxford University Press, New York (1994).

[34]. http://www.cvmob.ufba.br/.

[35]. N. Peña, B.C. Credidio, L.P.N.R.M.S. Corrêa, L.G.S. França, M.V. Cunha, M.C. Sousa, J.P.B.C. Vieira, J.G.V. Miranda. Free instrument for measurements of motion. Rev. Bras. Ensino Fís., 35 (3) (2013), pp. $1-5$.

[36]. A. Malinvemo. A Simple Method to Estimate the Fractal Dimension of a Self-Affine Series. Geophys. Res. Lett., 17 (11) (1990), pp. 1953-1956.

[37]. J.A. Durlak. How to select, calculate, and interpret effect sizes. J. Pediatr. Psychol. (2009) jsp004.

[38]. G.M. Sullivan, R. Feinn. Using effect size — or why the PP-value is not enough. J. Grad. Med. Educ., 4 (3) (2012), pp. 279-282.

[39]. M. Sekine, M. Akay, T. Tamura, Y. Higashi, T. Fujimoto. Fractal dynamics of body motion in patients with Parkinson's disease. J. Neural Eng., 1 (1) (2004), pp. 8-15.

[40]. O.E. Dick, S.P. Romanov, A.D. Nozdrachev. Energy and fractal characteristics of physiological and pathological tremors of the human hand. Hum. Physiol., 36 (2) (2010), pp. 203-210.

[41]. T. Herman, A. Weiss, M. Brozgol, N. Giladi, J.M. Hausdorff. Gait and balance in Parkinson's disease subtypes: objective measures and classification considerations. Journal of neurology, 261 (12) (2014), pp. 2401-2410.

[42]. J.M. Hausdorff, S.L. Mitchell, R. Firtion, C.K. Peng, M.E. Cudkowicz, J.Y. Wei, A.L. Goldberger. Altered fractal dynamics of gait: reduced stride-interval correlations with ageing and Huntington's disease. J. Appl. Physiol., 82 (1) (1997), pp. 262-269. 